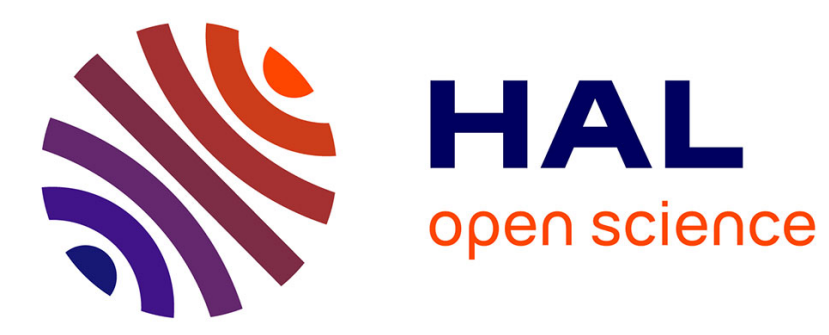

\title{
Bound on the counting function for the eigenvalues of an infinite multistratified acoustic strip
}

Olivier Poisson

\section{To cite this version:}

Olivier Poisson. Bound on the counting function for the eigenvalues of an infinite multistratified acoustic strip. 1998. hal-01429917

\section{HAL Id: hal-01429917 https://hal-amu.archives-ouvertes.fr/hal-01429917}

Preprint submitted on 9 Jan 2017

HAL is a multi-disciplinary open access archive for the deposit and dissemination of scientific research documents, whether they are published or not. The documents may come from teaching and research institutions in France or abroad, or from public or private research centers.
L'archive ouverte pluridisciplinaire HAL, est destinée au dépôt et à la diffusion de documents scientifiques de niveau recherche, publiés ou non, émanant des établissements d'enseignement et de recherche français ou étrangers, des laboratoires publics ou privés. 


\section{Bound on the counting function for the eigenvalues of an infinite multistratified acoustic strip \\ Olivier POISSON}

\section{MOS classification numbers}

35J20, 35L05, 35P, 47A70

\section{Summary}

Let $\mathcal{N}(\mu)$ be the counting function of the eigenvalues associated with the selfadjoint operator $-\nabla(\rho(x, z) \nabla \cdot)$ in the domain $\Omega=\mathbb{R} \times] 0, h[, h>0$, with Neuman or Dirichlet conditions at $z=0, z=h$. If $\rho=1$ in the exterior of a bounded rectangular region $\mathcal{O}$, that is, for $|x|$ large, then $\mathcal{N}(\mu)$ is known to be sublinear: the proof consists in the spectral analysis of a quadratic form obtained from a Green formula for $-\nabla(\rho(x, z) \nabla \cdot)$ on $\mathcal{O}$. In our case, the medium is multistratified: the function $\rho(x, z)$ satisfies $\rho(x, z)=\rho(z)$ for $|x|$ large. Since the direct use of the previous proof fails, we modify the quadratic form and obtain the estimate $N(\mu) \leq C \mu^{3 / 2}$. 


\section{Introduction and main results}

Consider the propagation of acoustic waves in a perturbed stratified medium described by the wave equation

$$
\frac{\partial^{2}}{\partial t^{2}} u(x, z, t)-\nabla(\rho(x, z) \nabla u(x, z, t))=0
$$

where $t \in \mathbb{R},(x, z) \in \Omega=\mathbb{R} \times] 0, h[, h>0$. The function $\rho(x, z)$ is the square of the celerity of acoustic waves in the strip $\Omega$. The asymptotic properties of $u(\cdot, x, z)$ for large $t$ can be derived from the spectral analysis of the self-adjoint operator $A$ defined by $A u:=-\nabla \cdot \rho(x, z) \nabla u$ with domain $D(A):=\{u \in$ $H_{\Omega} \mid A u \in L^{2}(\Omega)$ and $\left.\left(\rho \partial_{z} u\right)_{z=0}=0\right\}$ where $H_{\Omega}:=\left\{v \in \mathrm{H}^{1}(\Omega) \mid v(x, h)=0\right\}$ and $\mathrm{H}^{1}(\Omega)$ denotes the usual Sobolev space. In this way the waves satisfy a Neumann condition at $z=0$ and a Dirichlet condition at $z=h$.

The function $\rho(x, z)$ is real-valued, measurable and satisfies the following conditions: $\rho, \rho^{-1} \in L^{\infty}(\Omega)$ with $0<\rho_{\min } \leq \rho \leq \rho_{\max }<\infty$, and $\rho(x, z)=\rho_{ \pm \infty}(z)$ for $\pm x>M$ where $M \geq 0$. If $\rho(x, z)=\rho_{ \pm \infty}(z)$ almost everywhere in $\Omega$, the medium is said to be "unperturbed", the operator $A$ "free", and we then put $A_{ \pm}=A$. The spectrum $\sigma\left(A_{ \pm}\right)$of $A_{ \pm}$is well-known $[2,3,9]$. It is reduced to the essential spectrum $\sigma_{\text {ess }}\left(A_{ \pm}\right)=\left[S_{1}^{ \pm}\left(A_{ \pm}\right),+\infty[\right.$, where the number $S_{1}^{ \pm}\left(A_{ \pm}\right)$is the lower bound of $\mathcal{S}\left(A_{ \pm}\right)$, the discrete set of thresholds (see also $[2]$ ).

In the general case $A$ is considered as a perturbation of the free operators $A_{+}$and $A_{-}$coupled to each other. Thus the spectrum of $A$ consists of two parts. The first is the absolutely continuous spectrum $\sigma_{a c}(A)$ which coincides with the essential spectrum: $\sigma_{\text {ess }}(A)=\sigma_{\text {ess }}\left(A_{+}\right) \cup \sigma_{\text {ess }}\left(A_{-}\right)=\left[S_{1}(A),+\infty[\right.$, where $S_{1}(A):=\min \left(S_{1}^{+}\left(A_{+}\right), S_{1}^{-}\left(A_{-}\right)\right)$. The second, possibly void, is the point spectrum $\sigma_{p}(A) \subset\left[\rho_{\text {min }},+\infty\left[\right.\right.$. We prove that $\sigma_{p}(A)$ is a discrete set, and hence improve [9] where it is shown that the eigenvalues of $A$, counted with their multiplicity, cannot have a finite accumulation point, except maybe to the left at points of $\mathcal{S}\left(A_{-}\right) \cup \mathcal{S}\left(A_{+}\right)$. This last set is conveniently denoted by $\mathcal{S}(A)$ and called "the set of thresholds" for the operator $A$. In fact one needs to know the behaviour of the resolvent near the real axis and near thresholds. This question is partially solved by the limiting absorption principle developed in $[2,9]$, where the following Hilbert spaces equipped with obvious norms are introduced:

$$
\begin{aligned}
& L^{2, s}(\Omega)=\left\{u \in L_{l o c}^{2}(\Omega) \mid\left(1+x^{2}\right)^{\frac{s}{2}} u(x, z) \in L^{2}(\Omega)\right\} \\
& H^{1, s}(\Omega)=\left\{u \in L^{2, s}(\Omega) \mid \nabla u \in\left(L^{2, s}(\Omega)\right)^{2}\right\}
\end{aligned}
$$

for any real $s$. As proved in [9], the operator $(A-\zeta)^{-1}$ defined for $\zeta \in \mathbb{C}_{+}:=$ $\{\zeta \in \mathbb{C} \mid \Im m \zeta>0\}$ extends continuously to $\zeta=\mu \in Z^{C}:=\mathbb{R} \backslash(\mathcal{S}(A) \cup$ 
$\left.\sigma_{p}(A)\right)$ as an operator $R_{A}^{+}(\mu) \in B\left(L^{2, s}(\Omega), L^{2,-s}(\Omega)\right)$ which is equipped with the uniform topology of norms, for any $s>\frac{1}{2}$. The investigation of the analytic properties of $R_{A}^{+}(\cdot)$ is the next step to confirm that the point spectrum of $A$ is discrete. Consider now the counting function $\mathcal{N}_{A}(\mu):=\# \sigma_{p}(A) \cap[0, \mu]$. Estimates of $\mathcal{N}_{A}(\mu)$ are well-known when $\rho_{+\infty}(z)$ and $\rho_{-\infty}(z)$ are constant:

$$
\mathcal{N}_{A}(\mu) \leq C \mu+0\left(\mu^{\frac{1}{2}}\right) \text { as } \mu \rightarrow+\infty
$$

and the optimal value for $C$ is known (see $[6,8,1]$ ). To do this, note that if $\mu$ is an eigenvalue of $A$ with the eigenmode $\phi$, then $\mu$ is an eigenvalue of some operator $G(\mu)$, with the eigenmode $u=\phi_{\mid \mathcal{O}}$, restriction of $\phi$ to the domain $\mathcal{O}:=]-M, M[\times] 0, h\left[\left(^{1}\right)\right.$. The expression of $G(\mu)$ is obtained from a Green formula on $\mathcal{O}$ for $A$, using a Dirichlet-Neumann operator $T(\mu)$ (cf. section 2).

However, when the medium is really stratified, such a method fails. That is why we modify the operator $G(\mu)$ in section 3 . The problem is then more complicated, but we obtain the following estimate:

\section{Theorem 1.1}

$$
\mathcal{N}_{A}(\mu) \leq C^{*} \mu^{3 / 2}+0(\mu) \text { as } \mu \rightarrow+\infty
$$

where

$$
\mid \begin{array}{ll}
C^{*}:= & C_{+}^{*}+C_{-}^{*} \\
C_{ \pm}^{*}:= & \frac{1}{8} M \rho_{M, \min }^{-1} R_{\rho}^{ \pm} \rho_{ \pm \infty, m}^{-\frac{1}{2}} \\
\rho_{M, \min }:= & \text { inf ess } \rho(x, z) \text { in } \mathcal{O} \\
\rho_{ \pm \infty, M}:= & \text { sup ess } \rho_{ \pm \infty}(z) \\
R_{\rho}^{ \pm}: & \text {the lowest integer greater than or equal to } \sqrt{2}\left(\frac{\rho_{ \pm \infty, M}}{\rho_{ \pm \infty, m}}\right)^{2} \\
\rho_{ \pm \infty, m}:= & \text { inf ess } \rho_{ \pm \infty}(z) .
\end{array}
$$

In addition, the remainder $0(\mu)$ in (2) is bounded by $C \cdot \max (M, 1)(\mu+1)$ where $C$ does not depend on $M$.

This result still holds for any Dirichlet or Neumann boundary conditions at $z=0, z=h$.

The paper is composed of two parts. Section 2 is about the Dirichlet-Neumann operators $T(\zeta)$. In [9] it is proven that the mapping $T(\cdot)$ defined on $\overline{\mathbb{C}_{+}}$is continuous. We show here the analyticity of $T(\cdot)$ and give an explicit formula for $T^{\prime}(\mu)$.

In the second part, section 3, we prove that the point spectrum of $A$ is discrete. In fact by another method we are near to recovering some results of [4] about

\footnotetext{
${ }^{1}$ the case $M=0$ is trivial since $\sigma_{p}(A)$ happens to be void
} 
the meromorphic continuation of the resolvent of $A$ through the real axis, and we complete the proof in [9]. Then we prove theorem 1.1.

We shall use the following notations: $D_{t}$ denotes $\frac{\partial}{\partial i t}$ for the variable $t$, and $\|\cdot\|_{r, X}$ the usual norm of the Sobolev space $\mathrm{H}^{r}(X)$.

\section{The Dirichlet-Neumann operator}

\subsection{Definition of the thresholds}

There are two ways to "reduce" the operator $A_{ \pm}$and thus two ways to introduce the set of thresholds.

First let us consider the operator $A_{ \pm, z}:=-\frac{d}{d z}\left(\rho_{ \pm \infty} \frac{d}{d z} \cdot\right)$ self-adjoint on $L^{2}(] 0, h[)$, with domain $D^{ \pm}:=\left\{v \in H^{1}(] 0, h[) \mid A_{ \pm, z} v \in L^{2}(] 0, h[), \rho_{ \pm \infty} v^{\prime}(0)=\right.$ $v(h)=0\}$. It has compact resolvent and discrete spectrum which consists of positive eigenvalues: the thresholds $S_{1}^{ \pm}<S_{2}^{ \pm} \ldots<S_{n}^{ \pm} \cdots$.

It is more convenient to consider for real $\mu$ the self-adjoint operator $A_{ \pm, \text {red }}:=$ $-\left(\rho_{ \pm \infty}\right)^{-1}\left(\frac{d}{d z} \rho_{ \pm \infty} \frac{d}{d z}+\mu\right)$ on $L^{2}(] 0, h\left[, \rho_{ \pm \infty}(z) d z\right)$, with domain

$D_{ \pm, \text {red }}:=\left\{v \in H^{1}(] 0, h[) \mid A_{ \pm, \text {red }} v \in L^{2}(] 0, h[), \rho_{ \pm \infty} v^{\prime}(0)=v(h)=0\right\}$. Its spectrum is discrete and consists of an increasing sequence $\left\{K_{n}^{ \pm}(\mu)\right\}_{n>1}$ of eigenvalues, associated with an orthonormal basis $U_{n}^{ \pm}(\mu ; \cdot)$. In fact $K_{n}^{ \pm}(\mu)$ vanishes if and only if $\mu=S_{n}^{ \pm}$(cf. figure 1$)$.

We set $\mathcal{S}(A):=\mathcal{S}\left(A_{-}\right) \cup \mathcal{S}\left(A_{+}\right)$where $\mathcal{S}\left(A_{ \pm}\right)$denotes the set of thresholds of $A_{ \pm}$. By setting $\lambda^{\frac{1}{2}}$ the square root of $\lambda \in \mathbb{C}$ such that $\left.\arg \left(\lambda^{\frac{1}{2}}\right) \in\right]-$ $\pi / 2, \pi / 2]$, and by using the spectral representation of the operator $A_{ \pm, \text {red }}$, we can define the square root $A_{ \pm, r e d}^{\frac{1}{2}}$ of $A_{ \pm, \text {red }}$. The eigenvalues of $A_{ \pm, \text {red }}^{\frac{1}{2}}$ are $\sqrt{K_{n}^{ \pm}} \equiv i k_{n}^{ \pm}(\mu) \in i \mathbb{R}_{+}$for $1 \leq n \leq N^{ \pm}(\mu)$ and $\sqrt{K_{n}^{ \pm}} \equiv \theta_{n}^{ \pm}(\mu) \in \mathbb{R}_{+}$for $n>N^{ \pm}(\mu)$. We also put $k_{n}^{ \pm}:=i \theta_{n}^{ \pm}$for $n>N^{ \pm}$.

Let us define the bounded Dirichlet-Neumann operator $T^{ \pm}(\mu)$ from $\tilde{H}^{ \pm}:=$ $D\left(A_{ \pm, r e d}^{1 / 4}\right)\left(^{2}\right)$ into its antidual space $\tilde{H}^{ \pm^{\prime}}$ by:

$$
<T^{ \pm}(\mu) \varphi, \varphi>:=-\left(\varphi \mid A_{ \pm, r e d}^{\frac{1}{2}} \varphi\right)_{ \pm}=\sum_{n \geq 1} i k_{n}^{ \pm}\left|\varphi_{n}^{ \pm}\right|^{2}, \forall \varphi \in D\left(A_{ \pm, r e d}^{\frac{1}{2}}\right)
$$

where $(\cdot \mid \cdot)_{ \pm}$is the scalar product in $L^{2}(] 0, h\left[, \rho_{ \pm \infty}(z) d z\right)$ and $\varphi_{n}^{ \pm}:=\left(\varphi \mid U_{n}^{ \pm}\right)_{ \pm}$. One sets $T(\mu):=T^{-}(\mu) \oplus T^{+}(\mu)$ as a bounded operator from the Hilbert sum $\tilde{H}:=\tilde{H}^{-} \oplus \tilde{H}^{+}$into its antidual space $\tilde{H}^{\prime}$. By setting $\|\varphi\|_{\frac{1}{2}}:=<T(0) \varphi, \varphi>^{\frac{1}{2}}$, (resp. $\|\varphi\|_{-\frac{1}{2}}:=<\varphi, T(0)^{-1} \varphi>^{\frac{1}{2}}$ ), one defines a norm on $\tilde{H}$ (resp. on $\tilde{H}^{\prime}$, the antidual space of $\tilde{H}$ ) which does not depend on $\mu$. Note that the trace

\footnotetext{
${ }^{2}$ note that $\tilde{H}^{ \pm}$does not depend on $\mu$
} 
operator $\gamma$ is continuous and onto from $H_{o}:=\left\{v \in \mathrm{H}^{1}(\mathcal{O}) \mid v_{\mid z=h}=0\right\}$ into $\tilde{H}$ (cf. [9]).

Remark 2.1 Similar definitions hold for $A_{ \pm, \text {red }}$ with boundary conditions of Dirichlet or Neumann type at $z=0, z=h$.

\subsection{Characterization of $R_{A}^{+}(\mu)$ and $\operatorname{ker}(A-\mu)$}

It is usual to study $R_{A}^{+}$via the operator $T(\mu)$. In fact one has

Proposition 2.1 Let $\mu \in Z^{C}$, let $f \in L^{2}(\Omega)$ with support in $\overline{\mathcal{O}}$. Then the function $\phi:=R_{A}^{+}(\mu) f \in D(A)_{l o c} \cap L^{2,-s}(\Omega)$ is determined by:

$$
\phi(x, z)=\mid \begin{array}{ll}
W^{ \pm}(\mu) \gamma^{ \pm} u(|x|-M, z) & \text { for } \pm x>M \\
u(x, z) & \text { for }|x|<M .
\end{array}
$$

where

- $\gamma^{ \pm}$is the trace operator from $H_{o}$ into $\tilde{H}^{ \pm}$

- the operator $W^{ \pm}(\mu)$ is defined (for any real $\mu$ ) on $\tilde{H}^{ \pm}$by:

$$
W^{ \pm}(\mu) \varphi(x, z):=\sum_{n \geq 1} \varphi_{n} e^{i k_{n}^{ \pm} x} U_{n}^{ \pm}(z)
$$

- $u:=\phi_{\mid \mathcal{O}}$ is the unique $\left.{ }^{\beta}\right)$ solution in $H_{o}$ of the following variational problem:

$$
\forall v \in H_{o}, b(\mu ; u, v)=\int_{\mathcal{O}} f \bar{v} d x d z
$$

where for any $\mu \in \mathbb{R}, b(\mu ; \cdot, \cdot)$ is the continuous sesquilinear form on $H_{o} \times H_{o}$ :

$$
b(\mu ; u, v):=\int_{\mathcal{O}}\{\rho \nabla u \nabla \bar{v}-\mu u \bar{v}\} d x d z-<T(\mu) \gamma u, \gamma v>.
$$

The main results of [9] on the point spectrum of $A$ are resumed by

Proposition 2.2 Let $\mu \in \sigma_{p}(A) \cap\left[S_{N^{ \pm}}^{ \pm}, S_{N^{ \pm}+1}^{ \pm}[\right.$and $\phi \in D(A)$. Then the two following statements are equivalent:

1) $\phi$ does not vanish and $A \phi=\mu \phi$.

2) With the notations of proposition 2.1, $\phi$ is determined by the relations (4), where $u$ is a non-trivial solution of the homogenous problem (5) (i.e $f=0$ ), and $\varphi:=\gamma u$ satisfies $\varphi_{n}^{ \pm}=0$ for $1 \leq n \leq N^{ \pm}(\mu)$.

\footnotetext{
${ }^{3}$ because $\mu \notin Z$
} 
If the above conditions 1) and 2) hold, then $\mu$ and $u$ are associated eigenelements of the unbounded self-adjoint operator $G(\mu)$ on $L^{2}(\mathcal{O})$, characterized by the following quadratic form $Q(\mu)$ on $H_{o}$ :

$$
Q(\mu)(u):=\int_{\mathcal{O}} \rho|\nabla u|^{2} d x d z-<T_{R}(\mu) \gamma u, \gamma u>
$$

where $T_{R}(\mu)$ denotes the real part of $T(\mu): T_{R}(\mu):=\frac{1}{2}\left(T(\mu)+T(\mu)^{*}\right)$, and $T(\mu)^{*}$ the adjoint of $T(\mu)$.

This method is successful for a homogenous medium (i.e $\rho_{ \pm \infty}$ independent of $z$ ), in order to compute eigenvalues or to estimate $\mathcal{N}_{A}(\mu)$. Each eigenvalue $\lambda_{n}(\mu)$ of $G(\mu)$ is a function of $\mu$ whose regularity comes from that of $T(\cdot)$. Particularly with regard to analytic regularity. The same concerning the regularity of $R_{A}^{+}(\cdot)$. This is the interest of the following section.

\subsection{Analyticity of the family $\{T(\mu)\}_{\mu}$}

The main results of this part are theorems 2.2 and 2.3 which render precise the analytic continuation of $T(\cdot)$. Finally an explicit representation of the derivative $T^{\prime}(\mu)$ is given.

For the sake of simplicity, we assume that $A=A_{+}=A_{-}$and we suppress the indices + and - . In particular, we write $\tilde{H}$ and $\rho_{\infty}$ instead of $\tilde{H}^{ \pm}$and $\rho_{ \pm \infty}$. Setting $\left.\Omega^{+}:=\right] 0,+\infty[\times] 0, h[$ and defining $\gamma u$ as the trace of $u$ on $\Sigma:=\{0\} \times] 0, h[$, note that for any $u \in \mathrm{H}^{1}\left(\Omega^{+}\right)$satisfying $\gamma u=0$, the function $u$ can be uniquely extended to the Hilbert space $H_{i}^{1}$ defined by

$$
\begin{aligned}
& H_{i}^{1}:=L_{i}^{2} \cap \mathrm{H}^{1}(\Omega), \text { with } \\
& L_{i}^{2}:=\left\{u \in L^{2}(\Omega) \mid u(x, z)=-u(-x, z) \text { almost everywhere in } \Omega\right\} .
\end{aligned}
$$

This continuation will be still denoted by $u$.

For any fixed $\omega \in \mathbb{C}^{*}$ with $0<\arg (\omega)<\pi / 2$, one defines the following operator $B(\omega)$ with domain $D(\omega)$ :

$B(\omega):=\mathcal{B}(\omega) \equiv D_{z} \rho_{\infty}(z) D_{z}+\omega^{-2} \rho_{\infty}(z) D_{x}^{2}$ and

$D(\omega):=\left\{u \in H_{i}^{1} \mid \mathcal{B}(\omega) \in L_{i}^{2},\left(\rho_{\infty} D_{z} u\right)_{\mid z=0}=u_{\mid z=h}=0\right\}$. Note that if $u \in H_{i}^{1}$ then $\gamma u=0$. If in addition $u \in D(\omega)$, then $B(\omega) u \in L_{i}^{2}$. The operator $B(\omega)$ is closed and unbounded on $L_{i}^{2}$, but not symmetric. Its domain is dense in $L_{i}^{2}$, and we shall see in the proof of theorem 2.1) that it does not depend on $\omega$ : $D(\omega)=L_{i}^{2} \cap D(A)$.

Theorem 2.1 The resolvent set of $B(\omega)$ contains the domains $\mathbb{C}_{+}$and $\mathbb{R} \backslash \mathcal{S}(A)$.

\section{Proof}


Remark 2.2 The above proof shows that $\xi^{2} \hat{u} \in L^{2}(\Omega)$. In particular $D_{x}^{2} u$ and $D_{z} \rho_{\infty} D_{z} u$ belong to $L^{2}(\Omega)$. Thus $D(\omega)=L_{i}^{2} \cap D(A)$ is independent of $\omega$.

Let $\varphi \in \tilde{H}$, and $\mu \in \mathbb{R} \backslash \mathcal{S}(A)$. Let us consider the following vector $u_{\mu}$ in $\mathrm{H}^{1}\left(\Omega^{+}\right)$

$$
u_{\mu}(x, z):=\sum_{n \geq 1} \varphi_{n} e^{i k_{n}(\mu) \omega x} U_{n}(\mu ; z) \text { with } \varphi:=\sum_{n \geq 1} \varphi_{n}(\mu) U_{n}(\mu ; z) .
$$

One easily checks that $\mathcal{B}(\omega) u_{\mu}=\mu u_{\mu}$ in $L^{2}\left(\Omega^{+}\right)$, and

$$
\left(\rho_{\infty} \frac{\partial u_{\mu}}{\partial x}\right)_{\mid \Sigma}=\omega T(\mu) \varphi
$$

Here $T$ means $T^{+}$or $T^{-}$. Setting $v_{\mu}:=u_{\mu}-u_{0}$, one has $\gamma v_{\mu}=0$ and $(\mathcal{B}(\omega)-$ н) $v_{\mu}=\mu u_{0}$. The functions $u_{0}$ and $v_{\mu}$ being uniquely extended to $L_{i}^{2}$, one has:

$$
v_{\mu} \in D(\omega) \text { and } v_{\mu}=\mu(B(\omega)-\mu)^{-1} u_{0}
$$

which is analytic on $\mathbb{C}_{+}$according to $\mu$. Since $u_{\mu}=u_{0}+v_{\mu}$ and

$$
T(\mu) \varphi=T(0) \varphi+\omega^{-1}\left(\rho_{\infty} \frac{\partial v_{\mu}}{\partial x}\right)_{\mid \Sigma},
$$

one has

Theorem 2.2 The family $\{T(\mu)\}_{\mu}$ defined for $\mu \in \mathbb{R}$ admits an analytic continuation in $\overline{\mathbb{C}_{+}} \backslash \mathcal{S}(A)$. In addition, $T(\mu)-T(0)$ is a relatively compact perturbation of $T(0)$, since this operates from $\tilde{H}$ into itself (see remark 2.2).

Let us now define for $N \geq 1$ the operator $T^{N}(\mu) \in B\left(\tilde{H}, \tilde{H}^{\prime}\right)$ by:

$$
T^{N}(\mu) \varphi:=T(\mu) \varphi+ \begin{cases}-i k_{N}(\mu) \varphi_{N}(\mu) \rho_{\infty} U_{N}(\mu ; \cdot) & \text { if } \mu \geq S_{N} \\ \theta_{N}(\mu) \varphi_{N}(\mu) \rho_{\infty} U_{N}(\mu ; \cdot) & \text { if } \mu \leq S_{N}\end{cases}
$$

By applying the theory of Kato on analytic perturbations (cf. [7]) to the family of operators $A_{\text {red }}$, one proves that $U_{N}$ and $K_{N}$ are analytic in $\mu \in \mathbb{R}$. Thus $T^{N}$ is analytic on $\mathbb{R} \backslash \mathcal{S}(A)$, since $K_{N}$ never vanishes on this set. In addition there exists a complex domain $V$ containing $S_{N}$ such that $\left(T^{N}\right)_{\| S_{N-1}, S_{N}[}$ can be analytically extended onto $V \cap \overline{\mathbb{C}_{+}} \backslash\left[S_{N},+\infty\left[\right.\right.$ as an operator $T^{N}$, similarly to the function $\left(K_{N}\right)^{\frac{1}{2}}$. Because $\lim \theta_{N}(\zeta)=-i k_{N}(\mu)$, one can check that $\lim \tilde{T^{N}}(\zeta)=T^{N}(\mu)$, as $\left.\zeta \rightarrow \mu \in\right] S_{N}, S_{N+1}\left[\right.$, with $\zeta \in V \cap \mathbb{C}_{+}$. For $\mu \in \mathbb{R}$ consider the following characterization of the adjoint operator $T(\mu)^{*}$ of $T(\mu)$ :

$$
T(\mu)^{*} \varphi=-\sum_{1 \leq n \leq N} i k_{n}(\mu) \varphi_{n} \rho_{\infty} U_{n}(\mu ; \cdot)-\sum_{n>N} \theta_{n}(\mu) \varphi_{n} \rho_{\infty} U_{n}(\mu ; \cdot) .
$$


It admits an analytic continuation to $\mathbb{C}_{-}:=\left\{\zeta \in \mathbb{C} \mid \bar{\zeta} \in \mathbb{C}_{+}\right\}$, and $T(\zeta)^{*}=$ $\overline{T(\bar{\zeta})}$. It is then not hard to prove that $\left(T^{N}\right)_{\| S_{N-1}, S_{N}[}$ can be uniquely continued into $V^{\prime} \cap \overline{\mathbb{C}_{-}}$, where $V^{\prime}$ is some open complex domain containing $S_{N}$.

Because $\lim \theta_{N}(\zeta)=i k_{N}(\mu)$ as $\left.\zeta \rightarrow \mu \in\right] S_{N}, S_{N+1}\left[\right.$ with $\zeta \in V^{\prime} \cap \mathbb{C}_{-}$, one obtains under these contraints: $\lim \tilde{T^{N}}(\zeta)=T^{N}(\mu)$.

Choosing $V$ bounded, the operator $T^{N}$ is then analytic in $V \cap V^{\prime} \backslash\left\{S_{N}\right\}$ and bounded in $V$. Thus it is analytic in the neighbourhood of $S_{N}$. Let $\theta \in \mathbb{R}$, set $\mathcal{D}(\theta):=\left\{\zeta \in \mathbb{C} \mid \arg \left(\zeta-S_{N}\right)=\theta\right\}$, choose $a>0$ small enough to have $B_{a, N} \subset V \cap V^{\prime}$, and set $\mathcal{C}(\theta):=\left\{K_{N}(\zeta) \mid \zeta \in D(\theta) \cap B_{a, N}\right\}$. As the following estimate holds uniformly in $B_{a, N}$ :

$$
K_{N}(\zeta)=\left(S_{N}-\zeta\right)\left|K_{N}^{\prime}\left(S_{N}\right)\right|+0\left(\left|\zeta-S_{N}\right|^{2}\right)
$$

where $K_{N}^{\prime}\left(S_{N}\right)=-\left\|U_{N}\left(S_{N} ; .\right)\right\|_{0,0, h[}^{2}<0, \mathcal{C}(\theta)$ is then a cut in the set $K_{N}\left(B_{a, N}\right)$ and there exists an analytic determination of $K_{N}(\zeta)^{\frac{1}{2}}$ on $K_{N}\left(B_{a, N}\right) \backslash \mathcal{C}(\theta)$. This result completes theorem 2.2. Moreover one has

Theorem 2.3 The mapping $\zeta \rightarrow T(\zeta)$ defined for $\zeta \in \mathbb{C}_{+}$can be analytically continued into a neighbourhood of the real axis with branching points $S_{N}, N \geq$ 1. This analytic continuation has the following form:

$$
T(\zeta)=T_{N}(\zeta)+\sum_{n=1}^{N} \sqrt{\zeta-S_{n}} T_{1, n}(\zeta)
$$

where $\sqrt{\zeta-S_{n}}$ is defined by the condition $\sqrt{\zeta-S_{n}}>0$ for $\zeta>S_{n}$; the operators $T_{N}(\zeta)$ and $T_{1, n}(\zeta)(n \leq N)$ belong to $B\left(\tilde{H}, \tilde{H}^{\prime}\right)$, and the range of $T_{1, n}(\zeta)$ is one. For any integer $n$, the function $\zeta \rightarrow T_{1, n}$ is holomorphic in a neighbourhood $V_{n}$ of $\mathbb{R}$ and the function $\zeta \rightarrow T_{n}(\zeta)$ is holomorphic in $V_{n} \backslash\left[S_{n+1},+\infty[\right.$.

\section{Proof}

Let us set $T_{N}(\mu) \varphi:=\sum_{n>N} i k_{n} \varphi_{n} \rho_{\infty} U_{n}(\cdot)$ for $\mu \in \mathbb{R}, N \in \mathbb{N}$. The required property for $T_{N}$ comes from the properties of $T^{n}$ (defined by (7)) for $1 \leq n \leq$ $N$. The conclusion is straightforward.

Remark 2.3 If $\mu \in\left[S_{N}, S_{N+1}\left[\right.\right.$, then $T_{N}(\mu)$ coincides with the real part $T_{R}(\mu)$.

\subsection{Calculation of $T^{\prime}(\mu)$ :}

For $\mu, \lambda \in \mathbb{R}$, one has $(B(\omega)-\mu)\left(v_{\mu}-v_{\lambda}\right)=(\mu-\lambda) u_{\lambda}$. The derivative of $v_{\lambda}$ at $\lambda=\mu \in \mathbb{R} \backslash \mathcal{S}(A)$ is then:

$$
q_{\mu}:=\frac{d v_{\mu}}{d \mu}=(B(\omega)-\mu)^{-1} u_{\mu}\left(\text { where } u_{\mu} \in L_{i}^{2}\right) .
$$


This implies

$$
T^{\prime}(\mu) \varphi=\omega^{-1}\left(\rho_{\infty} \frac{\partial q_{\mu}}{\partial x}\right)_{\mid \Sigma} .
$$

Let us fix $\mu$ in $] S_{N}, S_{N+1}$ [, and suppress the corresponding indices to simplify: $u_{\mu}:=u, q_{\mu}:=q$ etc. Setting

$$
\tilde{u}(x, z):=\sum_{n \geq 1} \overline{\varphi_{n}} e^{i k_{n} \omega x} U_{n}(z)
$$

we get $\gamma \tilde{u}=\bar{\varphi},(\mathcal{B}(\omega)-\mu) \tilde{u}=0$. The Green formula

$$
\int_{\Omega^{+}} u \tilde{u} d x d z=\int_{\Omega^{+}}(B(\omega)-\mu) q \tilde{u} d x d z=\omega^{-2} \int_{\Sigma}\left(\rho_{\infty} \frac{\partial q}{\partial x}\right) \gamma \tilde{u} d z
$$

gives

$$
\left\langle T^{\prime}(\mu) \varphi, \varphi\right\rangle=\omega \int_{\Omega^{+}} u \tilde{u} d x d z .
$$

The last value, denoted by $J(\varphi)$, is independent of $\omega$. A short calculation gives

$$
J(\varphi)=-\sum_{n, m \geq 1} \frac{\varphi_{n} \overline{\varphi_{m}}}{i k_{n}+i k_{m}} a_{n, m} \text {, with } a_{n, m}:=\int_{0}^{h} U_{n}(z) U_{m}(z) d z
$$

In particular one has $\left\langle T_{N}^{\prime}(\mu) \varphi, \varphi\right\rangle=\Re e(J(\varphi))$ which is non-negative for $\varphi_{n}(\mu)=0,1 \leq n \leq N$. In fact in this case $\Re e(J(\varphi))$ is the square norm in $L^{2}\left(\Omega^{+}, d x d z\right)$ of the vector $\sum_{n>N} \varphi_{n} e^{-\theta_{n} x} U_{n}(z)$.

\section{Counting of the point spectrum of $A$}

\subsection{Absence of accumulation point of eigenvalues}

The following theorem proved by another method in [?] completes the result in $[9]$ :

Theorem 3.1 The point spectrum of $A$ is discrete.

The proof uses the non-negativity of $T(\mu)$ and $\Re e(J(\varphi))$ (see section 2).

Proof 


\subsection{Counting the eigenvalues of $A$}

This part is devoted to the proof of theorem 1.1, which is also valid for Dirichlet or Neumann boundary conditions. One denotes by $\mathcal{N}_{A}(\mu)$ the finite number of eigenvalues of $A$ (counted with their order of multiplicity) less or equal to $\mu$.

\section{Proof}

One proceeds in three steps. It is assumed until the second step that $\rho_{+\infty}=\rho_{-\infty}=: \rho_{\infty}$. The indices + and - are thus suppressed until we deal with the general case in the third step.

1. Setting $S_{0}:=0$, recall that (cf. section 2) if for some $N \geq 0, \mu \in$ $\left[S_{N}, S_{N+1}\right.$ [ is an eigenvalue of $A$ associated with the eigenmode $\phi$, then $u=\phi_{\mid \mathcal{O}}$ is a non-trivial solution in $H_{o}$ of the following equations:

$$
\forall v \in H_{o}, b(\mu ; u, v)=0
$$

and $(\gamma u)_{n}(\mu)$ is null for $1 \leq n \leq N$. Thus $(\mu, u)$ is a pair of eigenvalue and eigenmode for the unbounded self-adjoint operator $G(\mu)$ on $L^{2}(\mathcal{O})$, which is associated with the following quadratic form $Q(\mu)$ defined on $H_{o}$ :

$$
Q(\mu)(u):=\int_{\mathcal{O}} \rho|\nabla u|^{2} d x d z-<T_{R}(\mu) \gamma u, \gamma u>+t(\mu)(V(\mu) \gamma u \mid \gamma u)
$$

where $t(\mu)$ is an arbitrary real function, $V(\mu)$ is the finite range operator defined by $V(\mu) \varphi:=\sum_{n=1}^{N} \varphi_{n}(\mu) U_{n}(\mu ; \cdot)$, and $(\cdot \mid \cdot)$ denotes the scalar product in $L^{2}(] 0, h\left[, \rho_{\infty}(z) d z\right)$.

Let us consider a subdivision $0=\mu_{0}<\mu_{1}<\cdots<\mu_{k} \cdots$ of $\mathbb{R}_{+}$which contains the thresholds. The number of intervals $\left[\mu_{k}, \mu_{k+1}\right]$ contained in $\left[S_{n}, S_{n+1}\right]$ is $R_{n}$. On the interval $\left.\left.J_{k}:=\right] \mu_{k}, \mu_{k+1}\right] \subset\left[S_{N}, S_{N+1}\right]$, we choose a non-negative, differentiable, non-increasing function $t(\mu)$ satisfying

$$
\text { (i) } Q^{\prime}(\mu) \leq 0
$$

Lemma 3.1 Denoting by $\mathcal{N}_{A}(J)$ the number of eigenvalues of $A$ in the set $J \subset \mathbb{R}_{+}$, one has under condition (i):

$$
\mathcal{N}_{A}\left(J_{k}\right) \leq \rho_{M, \min }^{-1} C_{M} \mu_{k+1}+\max (M, 1) 0\left(\mu_{k+1}^{\frac{1}{2}}\right)
$$

where $C_{M}$ depends only on $M$ and the remainder $0\left(\mu_{k+1}^{\frac{1}{2}}\right)$ is independent of $M$.

\section{Proof}


Lemma 3.2 If the condition (i) is satisfied and if the sequence $\left\{R_{n}\right\}_{n}$ is bounded, then the required estimate holds:

$$
\mathcal{N}_{A}(\mu) \leq \frac{1}{2} R \rho_{M, \min }^{-1} \rho_{\infty, m}^{-\frac{1}{2}} C_{M} \mu^{3 / 2}+\max (M, 1) 0(\mu) \text { as } \mu \rightarrow+\infty .
$$

where $R$ is a bound for $\left\{R_{n}\right\}_{n}$ and the remainder $0(\mu)$ is independent of $M$.

\section{Proof}

2. On condition that we find the adequate subdivision $\left\{\mu_{k}\right\}$ and function $t(\mu)$, theorem 1.1 is proved.

Lemma 3.3 Let $\mu \in J_{k} \subset\left[S_{N}, S_{N+1}\right], \mu \notin \mathcal{S}(A)$. For any $u \in H^{1}(\mathcal{O})$ one has

$$
-Q^{\prime}(\mu)[u] \geq \rho_{\infty, M}^{-1} b^{2}-\left(C_{2, N}+C_{1, N} t(\mu)\right) a b-t^{\prime}(\mu) a^{2}
$$

with the notations:

$$
\begin{aligned}
a & :=\left(\sum_{n=1}^{N}\left|\varphi_{n}\right|^{2}\right)^{\frac{1}{2}} \\
b & :=\left(\sum_{m>N} \frac{\left|\varphi_{m}\right|^{2}}{\theta_{m}}\right)^{\frac{1}{2}} \\
\varphi & :=\gamma u \\
C_{1, N} & :=2 \rho_{\infty, m}^{-1} \rho_{\infty, M}^{3 / 4}\left(S_{N+1}-S_{N}\right)^{-3 / 4} \\
C_{2, N} & :=2 \sqrt{2} \rho_{\infty, m}^{-1} \rho_{\infty, M}^{1 / 4}\left(S_{N+1}-S_{N}\right)^{-1 / 4}
\end{aligned}
$$

\section{Proof}

Remark 3.1 Some additional calculations show that the numerical constant $1 / 8$ in (3) can be improved. However the estimates (2) on $C_{1, N}$ and $C_{2, N}$ are optimal. Putting $\varphi_{n}:=0$ if and only if $n \notin\{N, N+1\}$, this is easily checked.

Remark 3.2 The use of $C_{M}$ (instead of its present value $M / 4$ ) generalizes the results to a non-rectangular domain $\mathcal{O}$. In fact, the case of a non-rectilinear strip $\Omega$ can be treated too (cf. [5] for example). 


\section{References}

[1] T. CHRISTIANSEN. Private communication.

[2] E. CROC and Y. DERMENJIAN. Spectral analysis of a multistratified acoustic strip, part II: Asymptotic behaviour of acoustic waves in a stratified strip. SIAM J. Math. Anal., 27(6):1631-1652, 1996.

[3] E. CROC and V. IFTIMIE. Wave operators in a multistratified strip. I.E.O.T, To appear, 1998.

[4] Y. DERMENJIAN, O. POISSON, and B. VAINBERG. Resonances for multistratified acoustic waveguides. Applicable Analysis, 71:413-440, 1999.

[5] P. DUCLOS, P. EXNER, and P. STOVICEK. Curvature induced resonances in a two-dimensional dirichlet tube. Annales de l'I.H.P, Physique théorique, 62(1):81-101, 1995.

[6] D. S. JONES. The eigenvalues of $\Delta u+\lambda u=0$ when the boundary conditions are given on semi-infinite domains. Proc. Cambridge Soc., 49:668$684,1953$.

[7] T. KATO. Perturbation theory for linear operators. Springer-Verlag, Berlin, 1966.

[8] J. C. NEDELEC and F. STARLING. Integral equation methods in a quasiperiodic diffraction problem for the time harmonic Maxwell's equations. Technical Report 179, Ecole Polytechnique, C.M.A.P, 1988.

[9] O. POISSON. Study of the operator associated with the propagation of seismic waves in a stratified acoustic medium, Prépublication LATP 97-8, Université de Provence. 